\title{
Europawahl 2019: neue politische Konstellationen für die Wahlperiode 2019-2024
}

\author{
Rudolf Hrbek
}

This article argues that the results of the European elections 2019 differ considerably in the 28 member states of the European Union since they were primarily determined by factors within the respective national context: the priorities in the political discourse and the actual configuration of the party system. The article identifies new political constellations for the European Parliament (the formation and composition of party groups incl. the relative weight of national groups as well as the relations between party groups) and asks about their impact on the role of the European Parliament in the electoral period 2019-2024 and on the inter-institutional relations. The Spitzenkandidaten procedure serves as a first test case.

In seiner in dieser Zeitschrift erschienenen Analyse der Europawahl $2014^{1}$ hatte der Verfasser dieses Beitrags einleitend daran erinnert, dass im Vorfeld jener Wahl - der achten Direktwahl zum Europäischen Parlament - Einschätzungen zu vernehmen gewesen waren, wonach sich diese als Folge neuer Ausgangs- und Rahmenbedingungen (insbesondere die erstmalige Anwendung des Spitzenkandidaten-Verfahrens sowie das Erstarken euroskeptischer Stimmen in einer Reihe von Mitgliedstaaten) von den bisherigen sieben Wahlgängen signifikant unterscheiden würde. Es wurde angenommen, dass diese neuen Bedingungen Auswirkungen haben könnten: auf den Wahlkampf; auf die Resonanz, die sie bei den Medien und der Wählerschaft hervorrufen würden; auf die Wahlbeteiligung; auf den Charakter der Wahl als (erstmals) genuin europäischer, also in den Mitgliedstaaten als solcher wahrgenommener Vorgang und eben nicht als die bloße Summe „nationaler Sekundärwahlen“;2 schließlich und vor allem auf die Zusammensetzung des Europäischen Parlaments und deren Bedeutung für die interinstitutionellen Beziehungen mit der Europäischen Kommission, dem Europäischen Rat und dem Rat der Europäischen Union.

* Prof. em. Dr. Rudolf Hrbek, Institut für Politikwissenschaft, Eberhard Karls Universität Tübingen.

Der Beitrag wurde am 9. Juli 2019 abgeschlossen. Spätere Entwicklungen können entsprechend hier nicht aufgegriffen werden.

1 Rudolf Hrbek: Europawahl 2014: Kontinuität und neue Facetten, in: integration 3/2014, S. $205 \mathrm{ff}$.

2 Karlheinz Reif und Hermann Schmitt hatten diese These mit Blick auf die erste Direktwahl 1979 begründet (Nine Second-Order National Elections - A Conceptual Framework for the Analysis of European Election Results, in: European Journal of Political Research 1/1980, S. 3ff.). Hermann Schmitt hatte, 25 Jahre später, die These für die Europawahl 2004 überprüft (The European Parliament Elections of June 2004: Still SecondOrder?, in: West European Politics 3/2005, S. 650ff.) und kam zu folgenden Ergebnissen: (1) Die These gilt weiterhin, wenn auch nur in abgeschwächter Form, für Westeuropa, also die EU-15-Mitgliedstaaten; (2) für die neuen mittel- und osteuropäischen Mitgliedstaaten konnten dagegen nicht alle Hypothesen bestätigt werden, wofür vor allem das Fehlen konsolidierter Parteiensysteme verantwortlich sei. Ein gleichfalls differenziertes Bild ergab die Überprüfung der These für die Europawahl 2009 (Rudolf Hrbek: Europawahlen als „Second-Order National Elections“? Ein Paradigma im Licht der Europawahlen 2004 und 2009, in: Jürgen Mittag (Hrsg.): 30 Jahre Direktwahlen zum Europäischen Parlament (1979-2009). Europawahlen und EP in der Analyse, BadenBaden 2011, S. 63ff.) mit folgenden Ergebnissen: (1) Für bestimmte Ergebnisse und Merkmale der Wahlen sind eher spezifische Besonderheiten in einzelnen EU-Mitgliedstaaten verantwortlich, während die auf die These bezogenen Hypothesen nur teilweise bestätigt werden konnten; (2) die Unterschiede zwischen Westeuropa einerseits und Mittel- und Osteuropa andererseits haben sich abgeschwächt. 
Der Beitrag über die Europawahl 2014 hat - wie der Untertitel „Kontinuität und neue Facetten“ aufweist - keine signifikanten Unterschiede zu den sieben vorangegangenen Wahlen ausmachen können. Die Frage nach Kontinuität oder Wandel wurde aber mit Blick auf die Europawahl 2019 in ihrem Vorfeld erneut gestellt, wiederum unter Verweis auf ein ganzes Bündel von Rahmenbedingungen und die damit verbundenen Auswirkungen. Der folgende Beitrag wird unter anderem versuchen, eine Antwort auf diese Frage zu geben. Der Untertitel signalisiert, dass in der Europawahl 2019 ein Einschnitt für das Europäische Parlament und die weitere Entwicklung der Europäischen Union (EU) gesehen werden kann, dessen Bedeutung und Wirkung sich im Lauf der kommenden fünf Jahre zeigen werden. Faktoren für einen solchen Einschnitt sind mit den Rahmenbedingungen für die Europawahl 2019 verbunden, die im Folgenden kurz genannt und erläutert werden sollen.

\section{Rahmenbedingungen}

Auch bei den Wahlen zum Europäischen Parlament der neunten Legislaturperiode (2019-2024), die vom 23. bis 26. Mai 2019 abgehalten wurden, ${ }^{3}$ galt kein einheitliches europäisches Wahlrecht. Der Direktwahlakt vom 20. September 1976 gibt nur einen allgemeinen Rahmen für die jeweils nationalen Wahlgesetze der EU-Mitgliedstaaten vor. Eine 2002 vorgenommene Änderung bestimmte, dass in allen Mitgliedstaaten das Verhältniswahlrecht anzuwenden ist. ${ }^{4}$ Im Juli 2018 wurde der Direktwahlakt durch einen Beschluss des Rates und des Europäischen Parlaments erneut geändert. Wichtige Neuerungen bezogen sich, erstens, auf die Einführung einer verbindlichen Sperrklausel in Höhe von mindestens 2 Prozent und höchstens 5 Prozent für alle Mitgliedstaaten mit mehr als 35 Mandaten $^{5}$ und, zweitens, auf jeweils national zu bestimmende Sanktionen zur Vermeidung doppelter Stimmabgabe. ${ }^{6}$ Die neuen Bestimmungen können jedoch erst wirksam werden, wenn sie von allen Mitgliedstaaten bestätigt werden. Da dieser Prozess noch nicht abgeschlossen ist, werden sie erst bei den Wahlen 2024 in Kraft treten. ${ }^{7}$ Ein weiteres Element des neuen Direktwahlakts sollte die Einführung transnationaler Listen sein, über die 10 bis 15 Prozent der Mandate verteilt werden sollten. Der entsprechende Vorschlag fand im Europäischen Parlament allerdings keine Mehrheit. ${ }^{8}$

Ein zweiter Faktor bezieht sich auf die Frage, ob und in welcher Weise die mit dem Vertrag von Lissabon erfolgte signifikante Stärkung des Europäischen Parlaments, zu der vor

3 Nicht in allen Mitgliedstaaten ist der Sonntag der traditionelle Wahltag.

4 Beschluss des Rates vom 25. Juni 2002 und 23. September 2002 zur Änderung des Akts zur Einführung allgemeiner unmittelbarer Wahlen der Abgeordneten des Europäischen Parlaments im Anhang zum Beschluss 76/787/EGKS, EWG, Euratom, in: Amtsblatt der Europäischen Gemeinschaften, Nr. L 283 vom 21. Oktober 2002, S. $1 \mathrm{ff}$.

5 Diese Regel würde lediglich in Deutschland und Spanien Auswirkungen haben. Alle anderen Staaten mit mehr als 35 Mandaten haben bereits eine Sperrklausel.

6 Beschluss (EU, Euratom) 2018/994 des Rates vom 13. Juli 2018 zur Änderung des dem Beschluss 76/787/EGKS, EWG, Euratom des Rates vom 20. September 1976 beigefügten Akts zur Einführung allgemeiner unmittelbarer Wahlen der Mitglieder des Europäischen Parlaments, in: Amtsblatt der EU, Nr. L 178 vom 16. Juli 2018, S. $1 \mathrm{ff}$.

7 Aus diesem Grund trat in Deutschland bei der Europawahl 2019 - wie schon 2014 - nach zwei Urteilen des Bundesverfassungsgerichts aus den Jahren 2011 bzw. 2013 gegen eine Fünf-Prozent- bzw. eine Drei-ProzentKlausel eine größere Anzahl kleinerer und Kleinst-Gruppierungen mit eigenen Listen zur Wahl an, von denen sieben je ein Mandat und eine zwei Mandate gewonnen haben.

8 Ausschlaggebend dafür war die ursprünglich nicht erwartete Ablehnung durch die EVP-Fraktion. Hier wurde schließlich argumentiert, dass die WählerInnen die zur Wahl stehenden Kandidaten kennen müssten, was unter den jetzt noch gegebenen Umständen für Kandidaten auf transnationalen Listen nicht der Fall sei. 
allem das Mitentscheidungsrecht bei der Rechtsetzung gehört, in der zurückliegenden achten Wahlperiode (2014-2019) wahrgenommen und gewürdigt worden ist. So wurde Anfang 2019 bspw. sehr ausführlich über die Rolle des Europäischen Parlaments bei der Beschlussfassung über die Urheberschutz-Richtlinie berichtet.

Das 2014 erstmals angewandte Spitzenkandidaten-Verfahren, ein dritter Faktor, sollte auch bei der Wahl 2019 praktiziert werden. An das Verfahren wurde und wird auch diesmal eine Reihe von Erwartungen geknüpft: Die Personalisierung würde den Wahlkampf bereichern und die Wählerschaft zusätzlich dadurch mobilisieren, dass mit der Stimmabgabe für eine (nationale) Partei und damit zugunsten der entsprechenden europäischen politischen Partei, die der Wählerschaft einen Spitzenkandidaten präsentiert, direkter Einfluss auf die Vergabe eines Spitzenamtes - das des Präsidenten der Europäischen Kommission - verbunden ist. Damit würde auch die Wahlbeteiligung steigen. Und mit Kandidaten, die nicht nur als Persönlichkeiten, sondern mit jeweils eigenen programmatisch-inhaltlichen Vorstellungen konkurrieren, würde die Politisierung der Europapolitik gefördert, welche ebenfalls mobilisiert. Schließlich würde der mit diesem Verfahren verknüpfte transnationale Wahlkampf die Herausbildung eines europäischen öffentlichen Raums, als Voraussetzung für das Entstehen eines europäischen Demos, fördern. Auch wenn sich diese Erwartungen bei der erstmaligen Anwendung des Verfahrens 2014 nicht - oder allenfalls in bescheidenen Ansätzen - erfüllten, entschieden sich mehrere europäische politische Parteien dafür, dieses 2019 erneut anzuwenden und damit zu stabilisieren. Sieben europäische politische Parteien - mehr als $2014^{9}$ - präsentierten ihre Spitzenkandidaten. ${ }^{10}$

Die Zunahme EU-kritischer Stimmen, vor allem rechtspopulistischer Provenienz, ist ein vierter Faktor. Das Aufkommen rechtspopulistischer Parteien in einigen EU-Mitgliedstaaten ist zum Ersten eine Herausforderung für die betreffenden Staaten, denn es bedroht die Stabilität der jeweiligen Regierungen und beeinträchtigt damit auch deren europapolitische Handlungsfähigkeit. Zum Zweiten ist die von diesen politischen Kräften vehement vorgetragene Kritik an den EU-Institutionen (vorrangig der Kommission) und an der Pflicht zur Einhaltung spezifischer EU-Regeln (etwa der Stabilitätskriterien oder der Rechtsstaatlichkeit und Unabhängigkeit der Justiz), verbunden mit dem Insistieren auf die Berücksichtigung nationaler Interessen, eine Herausforderung für die EU und prägt auch den europapolitischen Diskurs im Rahmen des Wahlkampfs.

9 Die Allianz der Konservativen und Reformer in Europa (AKRE) hatte 2014 die Beteiligung an dem Verfahren noch abgelehnt.

10 Die Europäische Volkspartei (EVP) nominierte den Deutschen Manfred Weber (Christlich-Soziale Union, CSU), die Partei der Europäischen Sozialdemokraten/Sozialisten (PES) den Niederländer Frans Timmermans (Partij van de Arbeid, PvdA; Partei der Arbeit), die AKRE den Tschechen Jan Zahradil (Občanská demokratická strana, ODS; Demokratische Bürgerpartei), die Allianz der Liberalen und Demokraten für Europa (ALDE) ein aus sieben Personen bestehendes Team, dem auch die Dänin Margrethe Vestager (Radikale Venstre, RV; Radikale Linke) angehörte, die Europäische Grüne Partei (EGP) eine Doppelspitze mit der Deutschen Ska Keller (Bündnis 90/Die Grünen) und dem Niederländer Bas Eickhout (GroenLinks, GL; GrünLinks), die Europäische Freie Allianz (EFA) den Spanier Oriol Junqueras (Esquerra Republicana de Catalunya, ERC; Republikanische Linke Kataloniens) und die Europäische Linke (EL) eine Doppelspitze mit dem wallonischen Belgier Nico Cué (Parti du Travail de Belgique, PTB; Partei der Arbeit Belgiens) und der Slowenin Violeta Tomić (Levica). 
Ein fünfter Faktor ist die europapolitische Stimmungslage in den EU-Mitgliedstaaten, wie sie in Meinungsumfragen abgebildet wird. ${ }^{11}$ Danach beurteilen im April 201961 Prozent der Befragten die EU-Mitgliedschaft ihres Landes als gut, 68 Prozent sehen ausdrücklich Vorteile der Mitgliedschaft und ebenfalls 68 Prozent sprechen sich für den Verbleib in der EU aus. ${ }^{12}$ Als Hauptverdienste der EU werden Frieden und der freie Warenverkehr genannt. Besonders aufschlussreich ist die Veränderung zwischen 2014 und 2018 bei der Nennung von Hauptproblemen, die sich der EU stellen. Waren dies 2014 die wirtschaftliche und finanzielle Situation sowie die Arbeitslosigkeit, während Einwanderung, Klimawandel und Terrorismus nur geringe Werte erzielten, stellt sich die Situation Ende 2018 anders dar: Einwanderung wird mit deutlichem Abstand als drängendstes Problem bezeichnet, gefolgt von Terrorismus, der finanziellen und wirtschaftlichen Situation und dem Klimawandel. Das spiegelt sich auch in den Antworten auf die Frage, welche Themen im Wahlkampf vorrangig behandelt werden sollten. Besonders aufschlussreich ist das Datenmaterial aber hinsichtlich der Unterschiede, die zwischen den EU-Mitgliedstaaten bei der Nennung der Hauptprobleme bestehen: Wirtschaft und Wachstum rangieren in Zypern, Portugal, Italien, den drei baltischen Staaten, Polen, Rumänien, Bulgarien und Irland vorn; Klimaschutz steht für Schweden, Dänemark, Finnland, die Benelux-Staaten und Deutschland oben; Fragen der Sicherheit und Verteidigung sind in Finnland, den drei baltischen Staaten, Polen und Ungarn sehr wichtig; Einwanderung wird in Malta, Italien, Ungarn und Tschechien als vorrangiges Thema gesehen. ${ }^{13}$ Diese Diversität weist darauf hin, dass sich die Wählerschaft in den EU-Mitgliedstaaten und deren Abstimmungsverhalten primär an nationalen Belangen und Prioritäten orientieren dürften. Ein einheitliches Bild und ein klares Mandat für die Politik der EU in der Wahlperiode 2019-2024 lassen sich daraus nicht gewinnen.

Ein sechster Faktor ist der Verlauf des Brexit-Prozesses, wie dieser gesehen und bewertet wird und welche Schlussfolgerungen in anderen EU-Mitgliedstaaten daraus gezogen werden. Das betrifft die Frage möglicherweise sehr negativer Folgen eines Austritts aus oder auch nur einer Distanzierung von der EU und rückt die Frage nach den Vorteilen der EUMitgliedschaft in den Vordergrund.

Ein siebter Faktor bezieht sich auf die zeitliche Nähe der Europawahl zu nationalen Wahlen: Wenn solche nationalen Wahlen nur kurze Zeit zurückliegen, werden sich die konkurrierenden (nationalen) Parteien um eine Bestätigung bzw. um eine Korrektur des Kräfteverhältnisses auf nationaler Ebene bemühen; wenn nationale Wahlen bald auf die Europawahl folgen, kann diese von den Parteien als Test und Probelauf, also ebenfalls primär unter dem nationalen Blickwinkel, gesehen werden. In einzelnen EU-Mitgliedstaaten finden zeitgleich zur Europawahl andere Wahlen statt, wie z.B. in Belgien nationale Wahlen sowie Wahlen in den verschiedenen Gebietskörperschaften (Regionen, Provinzen, Kommunen). In Deutschland fanden zusammen mit der Europawahl in einigen Bundesländern Kommunalwahlen statt. Das kann, wie sich schon bei früheren Europawahlen zeigte, zu einer höheren Wahlbeteiligung führen, wenn nämlich die WählerInnen die je-

11 Emmanuel Rivière/Julien Zalc: The electoral context 2019: new and increasingly European issues, Fondation Robert Schuman: European Issue 513/2019. Das Policy Paper enthält interessante Daten aus Umfragen, auch im Vergleich zur Ausgangslage vor den Wahlen 2014.

12 Siehe die Eurobarometer-Umfragen, wie sie referiert werden in Rivière/Zalc: The electoral context 2019, 2019.

13 Ebenda. 
weils auch national anstehenden Wahlen, aus ganz unterschiedlichen subjektiven Beweggründen heraus, für besonders wichtig ansehen.

Und schließlich zeichnen sich in einer Reihe von EU-Mitgliedstaaten teils tiefgreifende Veränderungen in der Konfiguration des jeweiligen Parteiensystems ab - verursacht durch das Aufkommen ganz neuer oder durch den Niedergang etablierter Parteien. ${ }^{14}$ Die Parteien sehen die Europawahl dann als eine willkommene Gelegenheit und versuchen diese zu nutzen, um einen Aufwärtstrend zu bestätigen oder gar zu verstärken bzw. um einen Abwärtstrend zu stoppen oder zu korrigieren. In beiden Fällen stehen Belange der nationalen Politik im Mittelpunkt.

Jeder dieser Faktoren kann Auswirkungen auf verschiedene Aspekte der Europawahl haben, worauf im Folgenden zurückzukommen sein wird.

\section{Die Wahlbeteiligung}

Bei den ersten Direktwahlen 1979 in den damals neun Mitgliedstaaten der Europäischen Gemeinschaft lag die Wahlbeteiligung bei fast 62 Prozent. Sie ging bei den folgenden Wahlen - an ihnen war als Folge der Süd-, Nord- und Osterweiterung schrittweise eine immer größere Anzahl von Mitgliedstaaten beteiligt - stetig zurück und lag sowohl 2009 als auch 2014 bei knapp 43 Prozent. Auch wenn die Werte zwischen den Mitgliedstaaten erheblich differierten, wurde der Rückgang des Durchschnittswerts für die gesamte EU als Anzeichen für eine sinkende Zustimmung zur Politik der EU und zum Integrationsprojekt insgesamt interpretiert. Auffallend und mehr als nur ein Detail waren die teils signifikant niedrigen Werte in den neuen mittel- und osteuropäischen Mitgliedstaaten. Die niedrige Wahlbeteiligung stand in deutlichem Kontrast zu den Voten, die in den Beitrittsreferenden in diesen Staaten erreicht worden waren. Mangelnde Zustimmung zur EU und ihrer Politik konnte aber auch in zwei der Gründungsstaaten - in Frankreich und in den Niederlanden - registriert werden: Dort lag die Wahlbeteiligung unter dem Durchschnittswert und überdies wurden in beiden Staaten in den Referenden zum EU-Verfassungsvertrag keine Mehrheiten erreicht.

Vor diesem Hintergrund ist verständlich, dass die Höhe der Wahlbeteiligung im Jahr 2019 mit großer Spannung erwartet worden war. Das Ergebnis (siehe dazu Übersicht 1) wurde als Überraschung gewertet: Der Durchschnittswert lag mit knapp 51 Prozent um etwa acht Prozentpunkte über den jeweils bei den Wahlen 2009 und 2014 erzielten Werten. Der seit 1979 zu verzeichnende kontinuierliche Abwärtstrend scheint gebrochen. Die Frage nach den Gründen für das jetzige Gesamtergebnis und wie der Anstieg der Wahlbeteiligung zu bewerten ist, bedarf sorgfältiger Prüfung. Dafür ist der Blick auf einzelstaatliche Ergebnisse, die im Vergleich ein sehr uneinheitliches Bild vermitteln, erforderlich.

14 Beispiele dafür sind im Umfeld der Europawahl 2019 Frankreich, Italien und Spanien. Siehe hierzu auch Johannes Kohls/Manuel Müller: Die Wahlen zum Europäischen Parlament 2019: drei Ursachen für die Transformation im europäischen Parteiensystem, in diesem Heft, S. $218 \mathrm{ff}$. 
Übersicht 1: Wahlbeteiligung an den Europawahlen 1979-2019

\begin{tabular}{|c|c|c|c|c|c|c|c|c|c|c|c|c|c|c|}
\hline & 1979 & 1981 & 1984 & 1987 & 1989 & 1994 & $\begin{array}{l}1995 \\
1996\end{array}$ & 1999 & 2004 & 2007 & 2009 & 2013 & 2014 & 2019 \\
\hline $\mathrm{BE}$ & 91,36 & & 92,09 & & 90,73 & 90,66 & & 91,05 & 90,81 & & 90,39 & & 89,64 & 88,47 \\
\hline DE & 65,73 & & 56,76 & & 62,28 & 60,02 & & 45,19 & 43,00 & & 43,27 & & 48,10 & 61,38 \\
\hline FR & 60,71 & & 56,72 & & 48,80 & 52,71 & & 46,76 & 42,76 & & 40,63 & & 42,43 & 50,12 \\
\hline IT & 85,65 & & 82,47 & & 81,07 & 73,60 & & 69,76 & 71,72 & & 66,47 & & 57,22 & 54,50 \\
\hline LU & 88,91 & & 88,79 & & 87,39 & 88,55 & & 87,27 & 91,35 & & 90,76 & & 85,55 & 84,24 \\
\hline $\mathrm{NL}$ & 58,12 & & 50,88 & & 47,48 & 35,69 & & 30,02 & 39,26 & & 36,75 & & 37,32 & 41,93 \\
\hline DK & 47,82 & & 52,38 & & 46,17 & 52,92 & & 50,46 & 47,89 & & 59,54 & & 56,32 & 66,00 \\
\hline IE & 63,61 & & 47,56 & & 68,28 & 43,98 & & 50,21 & 58,58 & & 58,64 & & 52,44 & 49,70 \\
\hline UK & 32,35 & & 32,57 & & 36,37 & 36,43 & & 24,00 & 38,52 & & 34,70 & & 35,60 & 36,90 \\
\hline EL & & 81,48 & 80,59 & & 80,03 & 73,18 & & 70,25 & 63,22 & & 52,54 & & 59,97 & 58,69 \\
\hline PT & & & & 72,42 & 51,10 & 35,54 & & 39,93 & 38,60 & & 36,77 & & 33,67 & 30,75 \\
\hline ES & & & & 68,52 & 54,71 & 59,14 & & 63,05 & 45,14 & & 44,87 & & 43,81 & 60,73 \\
\hline SE & & & & & & & 41,63 & 38,84 & 37,85 & & 45,53 & & 51,07 & 55,27 \\
\hline AT & & & & & & & 67,73 & 49,40 & 42,43 & & 45,97 & & 45,39 & 59,80 \\
\hline FI & & & & & & & 57,60 & 30,14 & 39,43 & & 38,60 & & 39,10 & 40,70 \\
\hline $\mathrm{EE}$ & & & & & & & & & 26,83 & & 43,90 & & 36,52 & 37,60 \\
\hline LT & & & & & & & & & 48,38 & & 20,98 & & 47,35 & 53,48 \\
\hline LV & & & & & & & & & 41,34 & & 53,70 & & 30,24 & 33,53 \\
\hline ML & & & & & & & & & 82,39 & & 78,79 & & 74,80 & 72,70 \\
\hline PL & & & & & & & & & 20,87 & & 24,53 & & 23,83 & 45,68 \\
\hline SK & & & & & & & & & 16,97 & & 19,64 & & 13,05 & 22,74 \\
\hline SI & & & & & & & & & 28,35 & & 28,37 & & 24,55 & 28,89 \\
\hline $\mathrm{CZ}$ & & & & & & & & & 28,30 & & 28,22 & & 18,20 & 28,72 \\
\hline HU & & & & & & & & & 38,50 & & 36,31 & & 28,97 & 43,36 \\
\hline CY & & & & & & & & & 72,50 & & 59,40 & & 43,97 & 44,99 \\
\hline BG & & & & & & & & & & 29,22 & 38,99 & & 35,84 & 32,64 \\
\hline RO & & & & & & & & & & 29,47 & 27,67 & & 32,44 & 51,07 \\
\hline HR & & & & & & & & & & & & 20,84 & 25,24 & 29,86 \\
\hline Ges. & 61,99 & & 58,98 & & 58,41 & 56,67 & & 49,51 & 45,47 & & 42,97 & & 42,61 & 50,62 \\
\hline
\end{tabular}

BE: Belgien, DE: Deutschland, FR: Frankreich, IT: Italien, LU: Luxemburg, NL: Niederlande, DK: Dänemark, IE: Irland, UK: Vereinigtes Königreich, EL: Griechenland, PT: Portugal, ES: Spanien, SE: Schweden, AT: Österreich, FI: Finnland, EE: Estland, LT: Litauen, LV: Lettland, ML: Malta, PL: Polen, SK: Slowakei, SI: Slowenien, CZ: Tschechische Republik, HU: Ungarn, CY: Zypern, BG: Bulgarien, RO: Rumänien, HR: Kroatien, ges.: gesamt.

Quelle: Ergebnisse der Europawahl 2019. Wahlbeteiligung nach Jahr, 24. Juni 2019, abrufbar unter: https://europawahlergebnis.eu/wahlbeteiligung/(letzter Zugriff: 4.7.2019). 
Die außerordentlich hohen Werte in Belgien und Luxemburg stellen aufgrund der dort bestehenden Wahlpflicht Sonderfälle dar. ${ }^{15}$ In sechs Mitgliedstaaten ist im Vergleich zu 2014 die Beteiligung, entgegen dem überwiegenden Trend in der EU, leicht zurückgegangen: um ca. einen Prozentpunkt in Griechenland, um etwa zwei Prozentpunkte in Malta und um je ca. drei Prozentpunkte in Italien, Irland, Portugal und Bulgarien. Als Erklärung kann auf den europapolitischen Diskurs in diesen Staaten, aber auch auf ihre Erfahrungen mit EU-Politik (z.B. bei der Finanzkrise oder in Fragen der Migration) verwiesen werden. In den anderen EU-Mitgliedstaaten ist die Wahlbeteiligung, verglichen mit 2014, gestiegen. Dabei können, was das Ausmaß der Steigerung betrifft, zwei Gruppen von Staaten unterschieden werden: Bei den Staaten der einen Gruppe war der Anstieg zweistellig, bei den anderen fiel der Zuwachs geringer aus.

Einen hohen Anstieg, um knapp 17 Prozentpunkte, verzeichnete Spanien. Dies ist vor dem Hintergrund tief greifender Veränderungen in der Parteienlandschaft des Landes zu erklären, auf die die Parteien mit Mobilisierungsanstrengungen reagierten, die wiederum bei der Wählerschaft Wirkung erzielten. Der starke Anstieg in Polen (um ca. 22 Prozentpunkte) und Rumänien (um etwa 19 Prozentpunkte) ist das Ergebnis einer Polarisierung, bei der es um den Charakter nationaler politischer Strukturen, um die Beachtung konstitutioneller Prinzipien und um die Vorzeichen politischer Kultur geht. Zwei Staaten verzeichneten einen Anstieg von je knapp über 14 Prozentpunkten: in Österreich zu erklären als Reaktion auf das Zerbrechen der Regierungskoalition als Folge eines politischen Skandals; in Ungarn zu verstehen als Echo auf die von der dominierenden Partei in den Diskurs gebrachte nationalistische Rhetorik. Der Zuwachs um etwa 13 Prozentpunkte in Deutschland hat seine Hauptursache in den Verschiebungen im Stärkeverhältnis der Parteien, wie sie sich in den nach der Europawahl ermittelten Zahlen zur Wählerwanderung und in den Bemühungen der Parteien spiegeln, sich mittels verstärkter Mobilisierungsanstrengungen der Wählerschaft gegenüber auf diese Dynamik einzustellen. Der Anstieg um etwas mehr als zehn Prozentpunkte in Tschechien hat seinen Ursprung in den mit zunehmender Schärfe ausgetragenen Gegensätzen in einem fragmentierten Parteiensystem, was auch die Zunahme um knapp zehn Prozentpunkte in Dänemark erklärt. Die Spaltung der Gesellschaft, die sich zuletzt in der Zunahme gewaltsamer Proteste gegen als ungerecht empfundene soziale Verhältnisse manifestiert, sowie die Polarisierung zwischen zwei politischen Lagern im Vorfeld der nächsten Präsidentschaftswahlen erklären den Anstieg um knapp acht Prozentpunkte in Frankreich.

In all diesen Fällen waren es spezifische Gegebenheiten der jeweiligen nationalen Politik, die eine politische Dynamik ausgelöst haben, die zu einem deutlichen Anstieg der Wahlbeteiligung geführt hat, nicht aber Themen und Fragen der EU-Politik. Die höhere Wahlbeteiligung kann also nicht ohne Weiteres als ein Indiz für mehr Zustimmung zur EU und ihrer Politik interpretiert werden, zumal die bei den Mobilisierungskampagnen in manchen EU-Mitgliedstaaten vertretenen Argumente und Parolen ausgesprochen EU-kritisch waren. Im Vorfeld der Europawahl war deshalb auch ein Wahlergebnis erwartet worden,

15 Die Nichtbefolgung der Wahlpflicht wird seit längerem in beiden Staaten nicht mehr als Ordnungswidrigkeit sanktioniert. Dennoch ist die Wahlbeteiligung - wohl als Ergebnis gewohnheitsmäßigen Verhaltens - regelmäBig sehr hoch. 
das den Anteil EU-kritischer oder gar EU-feindlicher Kräfte im künftigen Europäischen Parlament erhöht.

\section{Zusammensetzung und Struktur des neu gewählten Europäischen Parlaments}

Im neu gewählten Europäischen Parlament der neunten Wahlperiode sind Abgeordnete aus 184 nationalen Parteien bzw. Listenverbindungen vertreten; das sind zwei weniger als bei der Europawahl 2014. Das neue Parlament ist jünger geworden: Der Altersdurchschnitt beträgt jetzt 51 Jahre im Vergleich zu 56 Jahren im Parlament der achten Wahlperiode 2014-2019. Drei Fraktionen liegen unterhalb dieser Durchschnittsmarke: Grüne/EFA ${ }^{16}$, $\mathrm{RE}^{17}$ und GUE/NGL ${ }^{18}$. Der Anteil von Frauen hat sich erneut erhöht: von 36 auf jetzt 40 Prozent, bei allerdings deutlichen Unterschieden zwischen den Fraktionen (Grüne/EFA 51 Prozent, RE 48 Prozent, GUE/NGL 45 Prozent, S\&D ${ }^{19} 43$ Prozent, ID ${ }^{20} 40$ Prozent, EVP ${ }^{21}$ 34 Prozent, EKR 2232 Prozent). Den höchsten Anteil weiblicher Abgeordneter weisen Schweden (55 Prozent) und Finnland (54 Prozent) auf; je 50 Prozent Frauen kommen aus Österreich, Malta, Slowenien, Frankreich, Lettland und Luxemburg; aus Deutschland sind es 36 Prozent; die Delegation aus Zypern besteht nur aus männlichen Abgeordneten. Die überwiegende Mehrheit der Abgeordneten der neunten Wahlperiode (435) wurde zum ersten Mal ins Europäische Parlament gewählt. Das bedeutet, dass sich diese Abgeordneten nun möglichst rasch mit den - festen und jahrelang bewährten - Strukturen und Arbeitsweisen des Parlaments vertraut machen müssen. Dafür ist die Zugehörigkeit zu einer Fraktion mit erfahrenen Mitgliedern sowie Mitarbeitern hilfreich. ${ }^{23}$

Die Gesamtzahl der Abgeordneten war gemäß Art. 14 Abs. 2 des Vertrags über die Europäische Union (EUV) bereits für die achte Wahlperiode auf 751 neu festgelegt worden. ${ }^{24}$ Die Bestimmung schreibt weiterhin vor, dass die BürgerInnen im Parlament degressiv proportional, mindestens jedoch mit sechs Mitgliedern pro Mitgliedstaat vertreten sind. Kein Mitgliedstaat erhält mehr als 96 Sitze. Mit Blick auf den Austritt des Vereinigten Königreichs aus der EU und den damit verbundenen Wegfall von 73 Mandaten hatten der Europäische Rat und das Europäische Parlament im Vorfeld des Brexits im Juni 2018 die künftige Zusammensetzung des Europäischen Parlaments wie folgt neu festgelegt: Die Gesamtzahl beträgt künftig 705 Abgeordnete; 27 der wegfallenden 73 Mandate werden unter Beachtung des Prinzips degressiver Proportionalität auf 14 unterrepräsentierte Mitgliedstaaten verteilt; die verbleibenden 46 Mandate werden nicht besetzt und können als Reserve für den Fall der Aufnahme neuer Mitgliedstaaten oder für eine Herabsetzung der Gesamtzahl der Abgeordneten verwendet werden. Übersicht 2 informiert über die Varianten der Zusammensetzung des Europäischen Parlaments.

16 Fraktion Die Grünen/Europäische Freie Allianz.

17 Fraktion „Renew Europe“ (ehemals Allianz der Liberalen und Demokraten für Europa, ALDE).

18 Fraktion Vereinigte Europäische Linke/Nordische Grüne Linke.

19 Fraktion der Progressiven Allianz der Sozialdemokraten im Europäischen Parlament.

20 Fraktion „Identität und Demokratie“ (ehemals Europa der Nationen und der Freiheit, ENF).

21 Fraktion der Europäischen Volkspartei.

22 Fraktion der Europäischen Konservativen und Reformisten.

23 Giulio Sabbati: Members of the European Parliament, 2019-2024, European Parliamentary Research Service: At a Glance, Infographic, Juli 2019.

24 Art. 14 Abs. 2 EUV schreibt dazu vor, dass der Europäische Rat einstimmig, auf Initiative des Europäischen Parlaments und mit dessen Zustimmung diesen Beschluss fasst. 
Übersicht 2: Sitzverteilung im Europäischen Parlament der neunten Wahlperiode (2019-2024)

\begin{tabular}{|c|c|c|c|c|c|c|c|c|}
\hline & $\begin{array}{l}\text { GUE/ } \\
\text { NGL }\end{array}$ & $\begin{array}{c}\text { Grüne/ } \\
\text { EFA }\end{array}$ & S\&D & $\begin{array}{c}\text { Renew } \\
\text { (ALDE) }\end{array}$ & EVP & EKR & $\begin{array}{c}\text { ID } \\
(\mathrm{ENF})\end{array}$ & f'los \\
\hline alt & 52 & 52 & 187 & 69 & 216 & 77 & 36 & $\begin{array}{l}20 \\
42 \text { (EFDD) }\end{array}$ \\
\hline $\begin{array}{l}\text { neu } \\
\text { (mit UK) }\end{array}$ & 41 & 75 & 154 & 108 & 182 & 62 & 73 & 56 \\
\hline $\begin{array}{l}\text { neu } \\
\text { (ohne UK) }\end{array}$ & 40 & 68 & 148 & 97 & 187 & 62 & 76 & 27 \\
\hline DE & $\begin{array}{l}5 \text { Linke } \\
1 \text { Tier }\end{array}$ & $\begin{array}{l}21 \text { Grüne } \\
1 \text { Piraten } \\
1 \text { ÖDP } \\
1 \text { Volt } \\
1 \text { Partei }\end{array}$ & $16 \mathrm{SPD}$ & $\begin{array}{l}5 \mathrm{FDP} \\
2 \mathrm{FW}\end{array}$ & 29 Union & 1 Familie & $11 \mathrm{AfD}$ & 1 Partei \\
\hline FR & $6 \mathrm{FI}$ & $\mathbf{1 2}^{+1} \mathrm{EELV}$ & $5^{+1}$ PS-PP & $21^{+2}$ LREM & $8 \mathrm{LR}$ & & $22^{+1} \mathrm{RN}$ & \\
\hline UK & $1 \mathrm{SF}$ & $\begin{array}{l}7 \text { Greens } \\
3 \text { SNP } \\
1 \text { PC }\end{array}$ & 10 Labour & $\begin{array}{l}16 \mathrm{LD} \\
1 \text { All }\end{array}$ & & 4 Cons & & $\begin{array}{l}29 \text { Brexit } \\
1 \text { DUP }\end{array}$ \\
\hline IT & & & $19 \mathrm{PD}$ & & $\begin{array}{l}6^{+1} \mathrm{FI} \\
1 \mathrm{SVP}\end{array}$ & $5^{+1} \mathrm{FdI}$ & $28^{+1}$ Lega & 14 M5S \\
\hline ES & $\begin{array}{l}5 \text { UP } \\
1 \text { Bildu }\end{array}$ & $\begin{array}{l}2 \mathrm{ERC} \\
1 \mathrm{CeC}\end{array}$ & $\mathbf{2 0}^{+1}$ PSOE & $\begin{array}{l}7^{+1} \text { C's } \\
1 \text { PNV }\end{array}$ & $12^{+1} \mathrm{PP}$ & $3^{+1}$ Vox & & $2^{+1} \mathrm{JxC}$ \\
\hline PL & & & $\begin{array}{l}5 \text { SLD } \\
3 \text { Wiosna }\end{array}$ & & $\begin{array}{l}14 \mathrm{PO} \\
3 \mathrm{PSL}\end{array}$ & $26^{+1} \mathrm{PiS}$ & & \\
\hline RO & & & \begin{tabular}{|l|}
$8^{+1}$ PSD \\
2 PRO
\end{tabular} & $\begin{array}{l}5 \text { USR } \\
3 \text { PLUS }\end{array}$ & \begin{tabular}{|l}
10 PNL \\
2 UDMR \\
2 PMP
\end{tabular} & & & \\
\hline NL & 1 PvdD & $3 \mathrm{GL}$ & 6 PvdA & \begin{tabular}{|l}
$4^{+1}$ VVD \\
2 D66
\end{tabular} & $\begin{array}{l}4 \text { CDA } \\
1 \text { 50plus } \\
1 \text { CU }\end{array}$ & $\begin{array}{l}3^{+1} \mathrm{FvD} \\
1 \text { SGP }\end{array}$ & $0^{+1}$ PVV & \\
\hline EL & 6 Syriza & & 2 KINAL & & $8 \mathrm{ND}$ & $1 \mathrm{EL}$ & & $\begin{array}{l}2 \mathrm{XA} \\
2 \mathrm{KKE}\end{array}$ \\
\hline BE & 1 PTB & $\begin{array}{l}1 \text { Groen } \\
2 \text { Ecolo }\end{array}$ & $\begin{array}{l}1 \text { sp.a } \\
2 \text { PS }\end{array}$ & $\begin{array}{l}2 \text { OpenVLD } \\
2 \mathrm{MR}\end{array}$ & $\begin{array}{l}2 \mathrm{CD} \& \mathrm{~V} \\
1 \mathrm{cdH} \\
1 \mathrm{CSP}\end{array}$ & $3 \mathrm{~N}-\mathrm{VA}$ & $3 \mathrm{VB}$ & \\
\hline PT & $\begin{array}{l}2 \mathrm{CDU} \\
2 \mathrm{BE}\end{array}$ & 1 PAN & 9 PS & & $\begin{array}{l}6 \text { PSD } \\
1 \text { CDS-PP }\end{array}$ & & & \\
\hline $\mathrm{CZ}$ & 1 KSČM & 3 Piráti & & 6 ANO & $\begin{array}{l}3 \text { TOP09 } \\
2 \text { KDU-ČSL }\end{array}$ & 4 ODS & 2 SPD & \\
\hline $\mathrm{HU}$ & & & $\begin{array}{l}4 \text { DK } \\
1 \text { MSZP }\end{array}$ & $2 \mathrm{MM}$ & 13 Fidesz & & & 1 Jobbik \\
\hline SE & $1 \mathrm{~V}$ & $2^{+1} \mathrm{MP}$ & $5 \mathrm{~S}$ & $\begin{array}{l}2 \mathrm{C} \\
1 \mathrm{~L}\end{array}$ & $\begin{array}{l}4 \mathrm{M} \\
2 \mathrm{KD}\end{array}$ & $3 \mathrm{SD}$ & & \\
\hline AT & & $2^{+1}$ Grüne & 5 SPÖ & $1 \mathrm{Neos}$ & 7 ÖVP & & 3 FPÖ & \\
\hline BG & & & 5 BSP & 3 DPS & $\begin{array}{l}6 \text { GERB } \\
1 \text { DSB }\end{array}$ & 2 WMRO & & \\
\hline DK & 1 Enhl. & $2 \mathrm{SF}$ & $3 S$ & \begin{tabular}{|l}
$3^{+1} \mathrm{~V}$ \\
$2 \mathrm{RV}$
\end{tabular} & $1 \mathrm{~K}$ & & $1 \mathrm{DF}$ & \\
\hline
\end{tabular}




\begin{tabular}{|c|c|c|c|c|c|c|c|c|}
\hline & $\begin{array}{l}\text { GUE} / \\
\text { NGL }\end{array}$ & $\begin{array}{l}\text { Grüne/ } \\
\text { EFA }\end{array}$ & S\&D & $\begin{array}{l}\text { Renew } \\
\text { (ALDE) }\end{array}$ & EVP & EKR & $\begin{array}{l}\text { ID } \\
(\mathrm{ENF})\end{array}$ & f'los \\
\hline FI & 1 Vas & $2^{+1}$ Vihr & $2 \mathrm{SDP}$ & $\begin{array}{l}2 \text { Kesk } \\
1 \text { SFP } \\
\end{array}$ & 3 Kok & & 2 PS & \\
\hline SK & & & 3 SMER & 2 PS & $\begin{array}{l}2 \text { Spolu } \\
1^{+1} \mathrm{KDH} \\
1 \text { OL }\end{array}$ & $2 \mathrm{SaS}$ & & 2 L'SNS \\
\hline IE & $\begin{array}{l}1 \mathrm{SF} \\
1 \text { parteilos } \\
2 \text { I4C }\end{array}$ & $2 \mathrm{GP}$ & & $1^{+1} \mathrm{FF}$ & $4^{+1} \mathrm{FG}$ & & & \\
\hline HR & & & $3^{+1} \mathrm{SDP}$ & 1 IDS & $4 \mathrm{HDZ}$ & $1 \mathrm{HKS}$ & & $\begin{array}{l}1 \text { parteilos } \\
1 \text { ŽZ }\end{array}$ \\
\hline LT & & 2 LVŽS & 2 LSDP & $\begin{array}{l}1 \mathrm{LRLS} \\
1 \mathrm{DP}\end{array}$ & $\begin{array}{l}3 \text { TS-LKD } \\
1 \text { parteilos }\end{array}$ & 1 LLRA & & \\
\hline LV & & 1 LKS & 2 SDPS & $1 \mathrm{AP} !$ & $2 \mathrm{~V}$ & $2 \mathrm{NA}$ & & \\
\hline SI & & & $2 \mathrm{SD}$ & 2 LMŠ & $\begin{array}{l}3 \text { SDS-SLS } \\
1 \text { NSi }\end{array}$ & & & \\
\hline EE & & & $2 \mathrm{SDE}$ & $\begin{array}{l}1 \mathrm{KE} \\
2 \mathrm{RE}\end{array}$ & $\mathbf{0}^{+1}$ Isamaa & & 1 EKRE & \\
\hline CY & 2 AKEL & & $\begin{array}{l}1 \text { DIKO } \\
1 \text { EDEK }\end{array}$ & & 2 DISY & & & \\
\hline LU & & 1 Déi Gréng & 1 LSAP & $2 \mathrm{DP}$ & $2 \mathrm{CSV}$ & & & \\
\hline MT & & & 4 PL & & $2 \mathrm{PN}$ & & & \\
\hline \multicolumn{9}{|c|}{$\begin{array}{l}\text { Die Darstellung in der Tabelle ist leicht vereinfacht. Abgeordnete von Kleinparteien oder parteilose Abgeord- } \\
\text { nete, die auf der Liste einer größeren Partei gewählt wurden, wurden gegebenenfalls dieser zugeordnet. }\end{array}$} \\
\hline \multicolumn{9}{|c|}{$\begin{array}{l}\text { Die Tabelle entspricht der Stärke und Fraktionszuordnung der Parteien zur Konstituierung des Parlaments. } \\
\text { Die Angaben }{ }^{\prime}+1^{\prime \prime} \text { oder }{ }^{\prime}+2^{\prime \prime} \text { beziehen sich auf die zusätzlichen Sitze nach dem britischen EU-Austritt. Zum } \\
\text { Beispiel bedeutet die Angabe "21 } 21^{2 \prime \prime} \text { bei der französischen LREM, dass die Partei zur Konstituierung des Parla- } \\
\text { ments } 21 \text { Sitze hatte und nach dem Brexit } 23 \text { haben wird. }\end{array}$} \\
\hline \multicolumn{9}{|c|}{$\begin{array}{l}\text { Die offizielle Sitzverteilung zum Zeitpunkt der Konstituierung am 2. Juli } 2019 \text { wich geringfügig von den hier } \\
\text { angegebenen Zahlen ab, da einige Sitze zu diesem Zeitpunkt vakant waren. Dies betrifft vor allem Situatio- } \\
\text { nen, in denen gewählte Europaabgeordnete kurz vor der Konstituierung des Parlaments auf ihr Mandat ver- } \\
\text { zichtet hatten und die Nachrücker noch nicht im Amt waren. Zudem befanden sich drei gewählte Abgeord- } \\
\text { nete aus Spanien ( } 2 \mathrm{JxC}, 1 \mathrm{ERC} \text { ) auf der Flucht oder im Gefängnis und konnten deshalb ihr Mandat nicht an- } \\
\text { treten. }\end{array}$} \\
\hline
\end{tabular}

Darstellung: Manuel Müller. Für weitere Details siehe Manuel Müller: Ergebnisse der Europawahl 2019, in:Der (europäische) Föderalist, abrufbar unter: https://www.foederalist.eu/p/ergebnisse-europawahl2019.html (letzter Zugriff: 15.7.2019).

Nach den diesjährigen Europawahlen wurden als erste wichtige interne Entscheidung Fraktionen gebildet. Dabei hatte sich bereits vor den Wahlen abgezeichnet, dass sich die Fraktionsstruktur hinsichtlich politischer Ausrichtung und Profil dieser Gruppierungen gegenüber der vorherigen achten Wahlperiode ${ }^{25}$ ändern würde, u.a. weil einige Parteien, deren Abgeordnete der ENF-Fraktion angehörten, angekündigt hatten, künftig eine neue Formation bilden zu wollen. Eine Fraktion kann laut Geschäftsordnung des Europäischen

25 Nach den Wahlen 2014 hatten sich zunächst sieben Fraktionen gebildet, siehe dazu die Übersicht bei Hrbek: Europawahl 2014, 2014, S. 218. Im Lauf der Wahlperiode kam es zur Bildung einer achten Fraktion, der ENF. Sie war mit 36 Mitgliedern die kleinste Fraktion. Ihr gehörten Mitglieder rechtspopulistischer Parteien wie Vlaams Belang (VB, Belgien), Front National (FN) bzw. Rassemblement National (RN, Frankreich), Lega (Italien), Partij voor de Vrijheid (PVV, Niederlande) und der Freiheitlichen Partei Österreichs (FPÖ, Österreich) an. 
Parlaments gebildet werden, wenn ihr mindestens 25 Abgeordnete angehören, die aus mindestens einem Viertel der Mitgliedstaaten - gegenwärtig sind das sieben - kommen.

Im neuen Europäischen Parlament haben sich sieben Fraktionen gebildet (siehe Übersicht 2). Fünf von ihnen treten mit demselben Namen wie bisher an und stehen insofern für Kontinuität: EVP, S\&D, Grüne/EFA, EKR und GUE/NGL. Die bisherige Fraktion ALDE hat im Zusammenhang mit dem Beitritt einer neu gegründeten großen französischen Partei $\left(\mathrm{LREM}^{26}\right)$ ihren Namen geändert und firmiert jetzt unter RE („Renew Europe“). Die siebte Fraktion ist eine Neugründung mit dem Namen „Identität und Demokratie“ (ID), in der sich rechtspopulistische Parteien zusammengefunden haben.

Wie schon in früheren Wahlperioden bleiben einige Abgeordnete (gegenwärtig 56 und nach vollzogenem Brexit 27) - zunächst - fraktionslos. Die beiden größten Kontingente in dieser Kategorie stellen die 29 Abgeordneten der neuen britischen Partei „Brexit“27 sowie die 14 Abgeordneten der italienischen Partei $\mathrm{M}_{5} \mathrm{~S}^{28}$. Weil mit dem Status als Fraktion die Verfügung über personelle, sächliche und räumliche Ressourcen sowie über Redezeit verbunden ist, bleibt abzuwarten, ob die Abgeordneten der M5S fraktionslos bleiben. Für die Abgeordneten der Partei „Brexit“ erledigt sich diese Frage mit vollzogenem Brexit automatisch.

Die Fraktionsstruktur des neuen Parlaments weist, verglichen mit der des Parlaments der achten Wahlperiode, eine in mehrfacher Hinsicht folgenreiche Veränderung auf: Die Fraktionen der EVP und der S\&D bleiben zwar die beiden stärksten Gruppierungen. Sie verfügen aber nun mit etwa 45 Prozent nicht mehr über die absolute Mehrheit, um bestimmte Fragen - darunter auch solche der Ämterbesetzung - allein zu entscheiden. Künftig sind mindestens drei oder vier Fraktionen - dabei immer vorausgesetzt, sie stimmen jeweils geschlossen, also ohne Abweichler, ab - erforderlich, um eine absolute Mehrheit zu bilden.

Absprachen, die das ermöglichen, werden diffiziler werden, weil die Anzahl der Beteiligten und damit auch die politische Diversität größer sind. Es gab schon bisher keine festen Bündnisse oder gar formalisierte Koalitionen, sondern die Fraktionen fanden sich bei Sachentscheidungen - je nach Politikfeld und einzelnem Dossier - in ganz unterschiedlichen Konstellationen zusammen, was teils schwierige und langwierige Aushandlungsprozesse erforderlich machte. Die Handlungsfähigkeit des Parlaments als Unionsorgan mit deutlich erweiterten und verstärkten Kompetenzen blieb dabei aber in aller Regel stets gewahrt. Bei veränderter Fraktionsstruktur wird es in Zukunft nicht einfacher sein, diese Handlungsfähigkeit durch Bildung der jeweils erforderlichen Mehrheiten zu gewährleisten.

Die zahlenmäßige Größe der Fraktionen und daran gemessen ihre Reihenfolge haben sich verändert. Die EVP bleibt mit 182 (bisher 216) Mitgliedern stärkste Fraktion, gefolgt von der S\&D mit 154 (bisher 187) Mitgliedern. Drittstärkste Fraktion ist die RE (bisher ALDE) mit 108 (bisher 69) Mitgliedern. Ihr folgen zwei fast gleich große Fraktionen: Die Fraktion Grüne/EFA hat mit 75 (bisher 52) Mitgliedern stark zugenommen; die neue Fraktion ID mit 73 Mitgliedern (hervorgegangen aus der bisherigen ENF-Fraktion mit nur 36

26 La République En Marche („Die Republik in Bewegung“).

27 Die Partei wurde von Nigel Farage, dem Vorsitzenden der früheren, jetzt nicht mehr bestehenden Partei UKIP (UK Independence Party), der für diese Partei auch dem bisherigen Europäischen Parlament angehört hatte, gegründet.

28 Movimento 5 Stelle (Fünf-Sterne-Bewegung). 
Mitgliedern) konnte ihre Erwartung, zur drittstärksten Kraft zu werden, nicht erfüllen. Die EKR-Fraktion, bisher drittstärkste Gruppe, folgt mit jetzt 62 (früher 77) Mitgliedern auf dem sechsten Platz, dahinter die Fraktion GUE/NGL mit nur 41 (bisher 52) Mitgliedern. Aus der Übersicht 2 ist weiterhin zu entnehmen, dass nach dem Ausscheiden des Vereinigten Königreichs zwei der sieben Fraktionen (EVP und ID) größer werden, die EKR ihre jetzige Größe beibehält, während die restlichen vier Fraktionen kleiner werden. ${ }^{29} \mathrm{Ob}$ und, wenn ja, wie sich diese Verschiebungen auf die praktische Arbeit des Europäischen Parlaments auswirken und was das für Bemühungen um die Bildung von (absoluten) Mehrheiten bedeutet, lässt sich zu Beginn der Wahlperiode nicht einschätzen.

Auch innerhalb der Fraktionen sind, als Ergebnis von teils gravierenden Verschiebungen in den nationalen Parteiensystemen, Veränderungen zu verzeichnen. Sie betreffen die Gesamtzahl der Fraktionsmitglieder, also die eben bereits erwähnte relative Stärke der Fraktionen, vor allem aber das Gewicht nationaler Delegationen in den Fraktionen.

Die Fraktion der EVP ist zwar die stärkste Gruppierung geblieben, die Zahl ihrer Mitglieder (sie kommen aus allen Mitgliedstaaten mit Ausnahme des Vereinigten Königreichs) hat sich aber reduziert. Das ist in erster Linie auf teils gravierende Verluste von Mitgliedsparteien in großen Mitgliedstaaten - wie Frankreich und Italien (jeweils minus etwa 60 Prozent), Polen und Spanien (jeweils minus etwa ein Drittel) sowie Deutschland (minus etwa 15 Prozent) - zurückzuführen. Wie stark das nationale politische und Parteiensystem die Wahlen beeinflusst haben, zeigen auch gegenläufige Tendenzen in anderen Staaten, in denen Mitgliedsparteien der EVP stärker geworden sind, wie etwa in Griechenland und Österreich, aber auch in Ungarn. Die weitere Mitgliedschaft von Abgeordneten der ungarischen Partei Fidesz in der Fraktion war aufgrund der Suspendierung von Mitgliedschaftsrechten von Fidesz in der europäischen Partei EVP zunächst unsicher. ${ }^{30}$ Als Fraktionsvorsitzender wurde der deutsche Abgeordnete Manfred Weber $\left(\mathrm{CSU}^{31}\right)$ wiedergewählt. ${ }^{32}$

Die Fraktion der S\&D bleibt, trotz starker Verluste, zweitstärkste Gruppierung. Die Einbußen gehen, ähnlich wie bei der EVP, auf die starken Verluste von Mitgliedsparteien in einigen großen Mitgliedstaaten zurück (in Deutschland und Italien ein Minus von jeweils mehr als 40 Prozent, in Frankreich ein Minus von mehr als 60 Prozent und in Rumänien ein Minus von 50 Prozent). Nach vollzogenem Brexit wird sich der Abstand der S\&D-Fraktion zur EVP-Fraktion deutlich vergrößern. Auch im Lager der sozialdemokratischen Parteienfamilie PES $^{33}$ gibt es, wie bei der EVP, gegenläufige Tendenzen. Gleichzeitig sind in einigen Staaten Parteien, die der PES angehören und deren Abgeordnete dann Mitglieder der S\&D-Fraktion sind, signifikant gestärkt worden: Das gilt vor allem für Spanien, in geringerem Ausmaß auch für die Niederlande und Portugal. Eine Kompensation der Ge-

29 Die Anzahl der fraktionslosen Abgeordneten wird sich nach dem erfolgten Brexit von 56 auf nur 27 in etwa halbieren.

30 Die mit Zustimmung von Fidesz beschlossene Suspendierung ihrer Mitgliedschaftsrechte in der europäischen Partei EVP und nicht ihr Ausschluss, den einzelne Mitglieder dieser europäischen Parteienfamilie gefordert hatten, zeigt den Nutzen, den sowohl Fidesz als auch die (große Mehrheit der) EVP im Verbleib dieser nationalen Mitgliedspartei im gemeinsamen Dachverband sehen. Für die EVP-Fraktion sind die jetzt 13 Fidesz-Abgeordneten eine quantitative Stärkung, für die ungarische Partei und ihre Abgeordneten bietet die Zugehörigkeit zur - überdies stärksten - Fraktion Vorteile.

31 Christlich-Soziale Union.

32 Diese Entscheidung erfolgte relativ bald nach der Europawahl, also bevor entschieden wurde, ob Weber als Spitzenkandidat der EVP das Amt des Präsidenten der Europäischen Kommission übertragen bekommt.

33 Partei der Europäischen Sozialdemokraten/Sozialisten. 
samtverluste der Fraktion war mit diesen Zuwächsen allerdings nicht möglich. Die fraktionsinterne Gewichtsverteilung hat sich indessen verschoben: Die Gruppe der PSOE-Abgeordneten $^{34}$ aus Spanien hat die deutsche SPD-Delegation ${ }^{35}$ als stärkste nationale Gruppe abgelöst. Zur Fraktionsvorsitzenden wurde dann auch die spanische Abgeordnete Iratxe García Pérez (PSOE) gewählt. Sie löst den bisherigen Vorsitzenden Udo Bullmann (SPD) $\mathrm{ab}$.

Drittstärkste Fraktion ist, mit 108 Abgeordneten, die Gruppierung mit dem neuen Namen „Renew Europe“ (RE). Das größte Kontingent in dieser Fraktion sind Abgeordnete aus Parteien, die der europäischen Partei ALDE angehören (auch die entsprechende Fraktion im Europäischen Parlament trug bislang diesen Namen). Im Kontext der letzten Präsidentschaftswahlen in Frankreich war auf Initiative und unter Leitung des Präsidentschaftskandidaten und späteren Wahlgewinners Emmanuel Macron eine neue, ganz auf ihn und sein nationales Reformprogramm ausgerichtete politische Partei, LREM, gegründet worden. Weil sie als einzelne nationale Partei mit ihren Abgeordneten im Europäischen Parlament keine Rolle spielen würde, erfolgten Sondierungen mit Parteien ähnlicher Ausrichtung (konkret: mit ALDE). Im Ergebnis gehören die 21 Abgeordneten von LREM jetzt als kleines Kontingent der Fraktion RE an. Diese musste dazu allerdings ihren bisherigen Namen ALDE aufgeben, da die mit dem Attribut „liberal“ verbundenen Assoziationen in Frankreich negativ besetzt waren und sind und als nicht vereinbar mit der traditionell starken Rolle des Staates (und der Regierung) bei der Steuerung des Wirtschaftsgeschehens gelten. Der Zusammenschluss mit den Abgeordneten der Partei LREM bedeutet für die Mitglieder der liberalen Parteienfamilie ALDE - ihre europäische politische Partei behält den bisherigen Namen - eine willkommene Stärkung ihrer Position im Europäischen Parlament. Der RE-Fraktion haben sich auch zwei neue, kleinere rumänische Parteien angeschlossen. Einer dieser Parteien $\left(\mathrm{PLUS}^{36}\right)$ gehört der zum Vorsitzenden der Fraktion gewählte Abgeordnete Dacian Cioloş an. ${ }^{37}$

Die in der achten Wahlperiode des Europäischen Parlaments drittstärkste Fraktion EKR ist als Folge des Einbruchs der britischen Conservative Party von 20 auf lediglich vier Mandate in der Rangfolge der Fraktionen in der neunten Wahlperiode auf den sechsten Platz zurückgefallen. Die Fraktion wird jetzt von Abgeordneten der polnischen Regierungspartei PiS $^{38}$ dominiert, die die Zahl ihrer Mandate von 19 auf 26 erhöhen konnte. In deutlichem Abstand folgen fünf Abgeordnete von $\mathrm{FdI}^{39}$, einer neuen rechtskonservativen italienischen Partei, sowie vier Abgeordnete der tschechischen Partei ODS ${ }^{40}$, die die Zahl ihrer Mandate verdoppeln konnte. Zu den Ko-Vorsitzenden der Fraktion wurden Ryszard Antoni Legutko (PiS) und Raffaele Fitto (FdI) gewählt.

Die Fraktion Grüne/EFA hat den 2014 erfolgten leichten Rückgang ihrer Mitgliederzahl mehr als wettgemacht: Sie steigerte ihre Mitgliederzahl um fast 50 Prozent und rangiert mit

34 Partido Socialista Obrero Español (Spanische Sozialistische Arbeiterpartei).

35 Sozialdemokratische Partei Deutschlands.

36 Partidul Libertăţii, Unităţii şi Solidarităţii (Partei der Einheit, Freiheit und Solidarität).

37 Es handelt sich um den seltenen Fall, dass das Mitglied einer kleinen Partei das Amt des Fraktionsvorsitzenden übernimmt.

38 Prawo i Sprawiedliwość („Recht und Gerechtigkeit“).

39 Fratelli d’Italia („Brüder Italiens“).

40 Občanská demokratická strana (Demokratische Bürgerpartei). 
75 Mandaten an vierter Stelle in der Rangliste der zahlenmäßig stärksten Fraktionen. Diesen Zuwachs verdankt die Fraktion vor allem dem starken Zuwachs der Grünen in Deutschland (fast eine Verdoppelung der Anzahl der Abgeordneten) und Frankreich (Verdoppelung der Anzahl der Mandate). Auch in Belgien und den Niederlanden legten die Grünen zu. Nicht zuletzt setzte sich die Verbreitung grüner Parteien in mittel- und osteuropäischen Mitgliedstaaten fort. Der Umstand, dass das Thema Klimaschutz bzw. Klimawandel in den Mitgliedstaaten auf breiter Front als vorrangig in und von der EU aufzugreifende Herausforderung eingestuft wird, hat für diesen Zuwachs grüner Parteien eine überragende Rolle gespielt. Untersuchungen zur Wählerwanderung in Deutschland haben sodann ergeben, dass die Grünen nicht nur bisherige WählerInnen aller anderen Parteien auf ihre Seite ziehen konnten, sondern dass sie auch bei der Mobilisierung bisheriger NichtwählerInnen erfolgreich waren. Grüne Parteien waren auch für Angehörige der jungen Generation überproportional attraktiv. Die Fraktion wird erneut von einer Doppelspitze - mit der Deutschen Ska Keller (Bündnis 90/Die Grünen) und dem Belgier Philippe Lamberts (von der wallonischen Partei Ecolo) - angeführt.

Die am linken Rand des politischen Spektrums angesiedelte Fraktion GUE/NGL, die, wie ihr Name verrät, ein relativ breites Bündnis von Parteien mit unterschiedlichen, stark im nationalen politischen und Parteiensystem verankerten Schwerpunkten darstellt, ging um etwa 20 Prozent geschwächt aus den Wahlen hervor und rangiert jetzt als kleinste Gruppierung. Für diese Schwächung waren vor allem die Verluste linker Parteien in Spanien (Halbierung der Abgeordnetenzahl) und Deutschland (Rückgang um etwa 40 Prozent) sowie in den Niederlanden und Tschechien (statt bisher je drei Mandaten konnten die Parteien hier nur je ein Mandat gewinnen). Zum Fraktionsvorsitzenden wurde der Deutsche Martin Schirdewan (Die Linke) gewählt. Die Fraktion vertritt EU-kritische Positionen und sie hat sich auch nicht am Spitzenkandidaten-Verfahren beteiligt.

In der neu gegründeten Fraktion ID, die im Europäischen Parlament so etwas wie die Nachfolgerin der mit nur 36 Mitgliedern sehr kleinen ENF-Fraktion darstellt, haben sich neben Parteien der bisherigen EFDD-Fraktion ${ }^{41}$ verschiedene am rechten Rand des politischen Spektrums angesiedelte Parteien versammelt. Hauptinitiator für die Bildung dieser Gruppierung - sie konstituierte sich als Fraktion am 13. Juni 2019 - war Matteo Salvini von der italienischen Lega-Partei, der sich schon im Vorfeld der Europawahl um eine solche Sammlungsbewegung EU-kritischer Parteien bemüht hatte. ${ }^{42}$ Die von ihm und seinen neuen Verbündeten geäußerte Erwartung, dass ihre neue Fraktion die drittstärkste Kraft im Europäischen Parlament werden würde, erwies sich als viel zu ambitiös. ID nimmt, noch hinter der Fraktion Grüne/EFA, den fünften Platz ein, mit deutlichem Abstand zu den drei größten Fraktionen EVP, S\&D und RE. Und auch das im Vorfeld der Wahl verfolgte Vorhaben, die ungarische Fidesz und die polnische PiS als Mitglieder in die Gruppierung einzubeziehen, blieb erfolglos. Der Fraktion gehören 73 Abgeordnete aus neun EUMitgliedstaaten an: ${ }^{43}$ Die größte nationale Gruppe besteht aus 28 Abgeordneten der italie-

41 Fraktion „Europa der Freiheit und der direkten Demokratie“.

42 Das Projekt einer neuen Fraktion, der Abgeordnete nationalkonservativer und rechtspopulistischer Parteien angehören sollten, wurde am 8. April 2019 in Mailand vorgestellt.

43 Die rechtspopulistische Partei PVV (Niederlande) mit ihrem Vorsitzenden Geert Wilders konnte kein Mandat gewinnen. Sie wird allerdings nach dem Brexit eines der drei dann an die Niederlande fallenden Mandate erhalten. 
nischen Lega, gefolgt von 22 Abgeordneten des französischen $\mathrm{RN}^{44}$ und 11 Abgeordneten der deutschen $\mathrm{AfD}^{45}$; kleinere Kontingente kommen aus Belgien $\left(\mathrm{VB}^{46}\right)$ und Österreich $\left(\mathrm{FPÖ}^{47}\right)$ mit je drei Mandaten, aus Finnland $\left(\mathrm{PS}^{48}\right)$ und Tschechien $\left(\mathrm{SPD}^{49}\right)$ mit je zwei Mandaten sowie aus Dänemark $\left(\mathrm{DF}^{50}\right)$ und Estland (EKRE $\left.{ }^{51}\right)$ mit je einem Mandat. Unübersehbar fehlt der Fraktion politische Homogenität: „Das Neinsagen ist der Kitt“. ${ }^{2}$ Bei einer Reihe wichtiger Sachfragen gibt es Gegensätze, ${ }^{53}$ was für das Wirken der Fraktion im Europäischen Parlament eine Hypothek darstellt. Zum Vorsitzenden der Fraktion wurde der Lega-Politiker Marco Zanni ${ }^{54}$ gewählt.

Die eben geschilderte politische Zusammensetzung des Europäischen Parlaments wirft eine Reihe von Fragen auf, die sich auf das künftige Verhältnis der Fraktionen zueinander, auf die interinstitutionellen Beziehungen zwischen dem Europäischen Parlament und dem Europäischen Rat sowie dem in jeweils unterschiedlichen Formationen auftretenden Rat und schließlich auf die Wahrnehmung der verschiedenen Kompetenzen und Funktionen des Parlaments beziehen. Was schon bisher galt, nämlich dass für Mehrheitsbildung und Gewährleistung der Handlungsfähigkeit des politisch fragmentierten Parlaments das Zusammenwirken von Fraktionen unerlässlich ist, gilt auch für das Parlament in der neunten Wahlperiode. Nur wird es künftig schwieriger werden, dass es zu diesem Zusammenwirken kommt. Je nach Konstellation müssen mindestens drei, wenn nicht vier Fraktionen einen gemeinsamen Nenner finden, ${ }^{55}$ denn die während früherer Wahlperioden vorhandene zahlenmäßige Mehrheit der beiden stärksten Fraktionen ist Vergangenheit. Wenn man zudem davon ausgeht, dass (1) sich die ID-Fraktion als dezidiert EU-kritisch definiert und angekündigt hat, häufig Nein zu sagen; dass (2) auch die EKR-Fraktion als EU-kritisch, wenngleich gemäßigter, gilt; und dass (3) schließlich die relativ kleine, zudem ein breites linkes Spektrum repräsentierende GUE/NGL-Fraktion zu vielen Fragen sehr grundsätzlich argumentiert und daher nur eingeschränkt kompromissbereit bzw. kompromissfähig ist, dann wird die Verantwortung für die Wahrnehmung der Funktionen des Europäischen Parlaments letztlich bei den vier anderen Fraktionen liegen, die über insgesamt 519 von 751 Stimmen (nach vollzogenem Brexit 500 von 705 Stimmen) verfügen. Sie werden der

44 Rassemblement National („Nationale Vereinigung“), bis Juni 2018 Front National.

45 Alternative für Deutschland.

46 Vlaams Belang („Flämische Interessen“).

47 Freiheitliche Partei Österreichs.

48 Perussuomalaiset („Wahre Finnen“).

49 Svoboda a prímá demokracie („Freiheit und direkte Demokratie“).

50 Dansk Folkeparti (Dänische Volkspartei).

51 Eesti Konservatiivne Rahvaerakond (Estnische Konservative Volkspartei).

52 So titelte der Kommentator in der Stuttgarter Zeitung am 14. Juni 2019. Und der AfD-Vorsitzende und Abgeordnete des Europäischen Parlaments Jörg Meuthen wird in einem dpa-Bericht (abgedruckt in Stuttgarter Zeitung vom 14. Juni 2019) mit den Worten zitiert: „Wir sind hierher gekommen, um Stachel im Fleisch der Eurokraten zu sein." Man werde oft Nein sagen, weil andere Parteien eine unsinnige Politik wollten. Das Ziel sei aber nicht „die Zerschlagung der EU“.

53 So etwa in der Frage einer national bestimmten Haushaltspolitik, wie sie die italienische Lega favorisiert, während die „Wahren Finnen“ eine zentrale Haushaltsüberwachung in einer Währungsunion für unerlässlich erklären. Ein anderes brisantes Thema betrifft die Sanktionen und die generelle Haltung gegenüber Russland. Das Angebot der österreichischen Regierung, Bewohnern Südtirols zusätzlich die österreichische Staatsangehörigkeit anzubieten, sorgt für Dissens zwischen Lega und der FPÖ.

54 Er hatte vorher der Partei M5S angehört.

55 Dabei wird unterstellt, dass die Fraktionen geschlossen agieren, was nicht als sicher angesehen werden kann. Es ist nicht ungewöhnlich, dass nationale Gruppen von Abgeordneten aus ganz unterschiedlichen Gründen von einem Fraktionsstandpunkt abweichen. 
Rolle und den konkreten Aktivitäten des Europäischen Parlaments in der neunten Wahlperiode das Gepräge geben.

\section{Das neu gewählte Europäische Parlament und die politisch-institutionelle Architektur der Europäischen Union}

Das Europäische Parlament ist, neben dem Europäischen Rat, zu Beginn einer Wahlperiode in Personalentscheidungen zur Besetzung von Ämtern in der Europäischen Kommission maßgeblich einbezogen. Die dafür einschlägigen Bestimmungen enthält Art. 17 Abs. 7 EUV, wobei sich die Sätze 1 bis 3 auf die Bestellung des Kommissionspräsidenten beziehen:

„Der Europäische Rat schlägt dem Europäischen Parlament nach entsprechenden Konsultationen mit qualifizierter Mehrheit einen Kandidaten für das Amt des Präsidenten der Kommission vor; dabei berücksichtigt er das Ergebnis der Wahlen zum Europäischen Parlament. Das Europäische Parlament wählt diesen Kandidaten mit der Mehrheit seiner Mitglieder. Erhält dieser Kandidat nicht die Mehrheit, so schlägt der Europäische Rat dem Europäischen Parlament innerhalb eines Monats mit qualifizierter Mehrheit einen neuen Kandidaten vor, für dessen Wahl das Europäische Parlament dasselbe Verfahren anwendet."

Die Bestimmungen gelten einem mehrstufigen komplexen Entscheidungsprozess, bei dem das Vorschlagsrecht des Europäischen Rates mit dem Wahlrecht des Europäischen Parlaments - jeweils mit bestimmten Mehrheiten - verbunden wird. Dem Europäischen Rat wird mit sehr ungenauen Formulierungen aufgegeben, (1) vor seinem Vorschlag „entsprechende Konsultationen“ durchzuführen und (2) dabei das Wahlergebnis zu „berücksichtigen".

2014 kamen diese Bestimmungen erstmals zur Anwendung, wobei einige der europäischen Parteien dem Entscheidungsprozess mit der lange vor der Wahl erfolgten Nominierung von "Spitzenkandidaten“ noch ein weiteres Element hinzugefügt hatten. Von einem solchen Element war im EUV keine Rede. Der Vertrag schloss ein solches politisches Element aber auch nicht aus. Diese Offenheit nutzten einige der europäischen politischen Parteien, um den Kreis der Kandidaten für das Amt des Kommissionspräsidenten zu begrenzen, damit also auch den Spielraum des Europäischen Rates hinsichtlich seines Vorschlagsrechts einzuengen.

Wie der Entscheidungsprozess 2014 dann tatsächlich ablief, ist ein Lehrstück für alle Beteiligten. ${ }^{56}$ Als das Wahlergebnis vorlag, wonach die EVP (mit ihrem Spitzenkandidaten Jean-Claude Juncker) ausreichend deutlich vor der S\&D (mit ihrem Spitzenkandidaten Martin Schulz) rangierte, nutzten diese zwei europäischen Parteien bzw. ihre jeweiligen Fraktionen im Europäischen Parlament - die dort über eine absolute Mehrheit verfügten die Offenheit der Vertragsbestimmungen bereits am Tag nach der Wahl zu einer weiteren politischen Initiative, nämlich zu einer Vorgabe für den weiteren Entscheidungsprozess zwischen dem Europäischen Parlament und dem Europäischen Rat: Letzterer wurde aufgefordert, Juncker als Kommissionspräsident vorzuschlagen. Mit dieser Aufforderung war das klare Signal verbunden, dass dieser Kandidat im Parlament die erforderliche Mehrheit finden, während ein anderer Kandidat durchfallen würde. Im Übrigen hätten auch die im

56 Vgl. dazu Hrbek: Europawahl 2014, 2014, S. 226. 
Vertrag vorgesehenen „entsprechenden Konsultationen“ durch den Europäischen Rat die Funktion gehabt zu eruieren, ob eine von der Mehrheit der Regierungen als Kandidat in Erwägung gezogene Persönlichkeit Chancen haben würde, die für die Wahl im Parlament erforderliche absolute Mehrheit zu gewinnen. Mit der raschen Aufforderung an die Regierungen war das Parlament - durch seine Mehrheit - einer Initiative des Europäischen Rates zuvorgekommen. Damit sei es, so wurde resümiert, gelungen, dem Wahlausgang eine unmittelbare politische Folgewirkung zu verleihen. Das neue Spitzenkandidaten-Verfahren, „ein Schritt ins europapolitisch Ungewisse“, habe seine erste Bewährungsprobe „geradezu glänzend bestanden “. ${ }^{57}$ Bekanntlich wurde Juncker dann auch vom Europäischen Rat vorgeschlagen ${ }^{58}$ und danach vom Europäischen Parlament gewählt.

Welche Schlussfolgerungen zogen die Beteiligten aus diesem Vorgang, der hier als „Lehrstück“ bezeichnet wird? Die europäischen politischen Parteien fühlten sich bestärkt, an dem Verfahren festzuhalten. Sie nominierten vor der Europawahl 2019, wie oben im Einzelnen aufgelistet, ihre Spitzenkandidaten, wobei sich diesmal auch die EKR beteiligte. Die Regierungen der meisten EU-Mitgliedstaaten dagegen stehen dem SpitzenkandidatenVerfahren skeptisch bis ablehnend gegenüber. Sie lehnen insbesondere seine Konstitutionalisierung strikt ab und haben wiederholt unterstrichen, dass die Aufstellung von Spitzenkandidaten durch die europäischen Parteien keinen Automatismus für das weitere Verfahren zur Bestellung des Präsidenten der Kommission zur Folge haben könne. Die Befürworter des Spitzenkandidaten-Verfahrens insistieren demgegenüber darauf, dass für das Amt des Kommissionspräsidenten nur eine Persönlichkeit infrage komme, die als Spitzenkandidat nominiert worden sei. Damit standen sich bei der Europawahl 2019 zwei nicht nur unterschiedliche, sondern letztlich unvereinbare Ausgangspositionen gegenüber.

Wie die maßgeblich Beteiligten mit dieser Situation umgegangen sind, kann durchaus als ein weiteres Lehrstück bezeichnet werden, mit schwerwiegenden und möglicherweise lange anhaltenden Auswirkungen auf die politisch-institutionelle Architektur der EU. Das Votum der WählerInnen hatte ein auf der einen Seite klares Ergebnis erbracht: Die EVPFraktion mit ihrem Spitzenkandidaten Weber rangierte an der Spitze, gefolgt von der S\&DFraktion mit ihrem Spitzenkandidaten Frans Timmermans und der RE-Fraktion, bei der die dänische Politikerin Margrethe Vestager - als Mitglied des Spitzenkandidaten-Teams von ALDE - mittlerweile als Kandidatin bevorzugt wurde. Während aber 2014 das Ergebnis $^{59}$ zur Folge hatte, dass sich beide Kandidaten und ihre Fraktionen ohne Verzug darauf verständigten, Juncker den Vorrang zu geben und sich auf seine Wahl zum Präsidenten der Kommission zu verpflichten, gab es diesmal eine solche Verständigung, in die mehr als nur zwei Fraktionen hätten einbezogen sein müssen, nicht. Das institutionelle Eigeninteresse des Europäischen Parlaments, sich gegenüber dem Europäischen Rat zu profilieren (es hätte von einer absoluten Mehrheit artikuliert werden müssen), trat gegenüber parteipolitischen Interessen und Ambitionen in einem politisch stärker fragmentierten Europäischen Parlament zurück. Timmermans, möglicherweise auch Vestager (und die hinter ihnen stehenden Parteien/Fraktionen) haben es offensichtlich für möglich gehalten, dass das $\mathrm{Zu}$ -

57 So Klaus Hänsch: Was erlauben Schulz?, in: Frankfurter Allgemeine Zeitung, 21. Juli 2014.

58 Der britische Premierminister David Cameron und der ungarische Ministerpräsident Viktor Orbán votierten beide gegen Juncker.

59 Die EVP mit Juncker rangierte vor der PES bzw. der S\&D-Fraktion mit Schulz. 
sammenwirken politischer Kräfte, ohne die EVP, bewirken könnte, dass er oder sie das Amt des/der Präsidenten/Präsidentin der Kommission übernehmen könnte - und eben nicht der Kandidat der EVP Weber. Zu diesem Kalkül haben sicherlich auch deutliche Signale aus dem Kreis der Mitglieder des Europäischen Rates beigetragen, wonach Weber mehrheitlich keine Unterstützung erfahren, ja von vielen vehement abgelehnt würde. Die wiederholt von einer Mehrheit im Europäischen Parlament vorgetragene Auffassung und Forderung, dass für das Amt des Präsidenten der Kommission nur eine Person infrage komme, die als Spitzenkandidat nominiert gewesen war, ohne dass sich eine Mehrheit gleichzeitig auf eine der infrage kommenden Personen geeinigt und diese Position dem Europäischen Rat übermittelt hätte, bedeutete, dass der Europäische Rat - zunächst allein - das Gesetz des Handelns übernehmen konnte.

Der Europäische Rat war zunächst auch nicht in der Lage, sich mit der erforderlichen Mehrheit auf einen Kandidaten zu verständigen. Die Staats- und Regierungschefs waren insgesamt gegen das Spitzenkandidaten-Verfahren und einen damit verbundenen Automatismus. Der französische Präsident Macron etwa lehnt einen entsprechend starken Einfluss seitens einer parlamentarischen Versammlung auf Kosten des unbedingten Vorrangs der Regierung ab. Dann gab es im Kreis der Mitglieder des Europäischen Rates Vorbehalte gegen einzelne Spitzenkandidaten, weil sie, wie etwa Weber, keine Erfahrungen aus einem Regierungsamt hätten oder weil sie sich, wie Timmermans, gegenüber einzelnen Mitgliedstaaten in Fragen der Beachtung und Einhaltung rechtsstaatlicher und demokratischer Prinzipien (zu) stark exponiert hätten.

Bei der Beurteilung des Aushandlungs- und Entscheidungsprozesses im Europäischen Rat darf aber nicht ausgeblendet oder vergessen werden, dass das Interesse seiner Mitglieder nicht allein auf die Besetzung des Amts des Kommissionspräsidenten gerichtet war. Tatsächlich standen Entscheidungen über die Besetzung mehrerer Spitzenämter an: des Präsidenten des Europäischen Rates, des Hohen Vertreters der Union für Außen- und Sicherheitspolitik, des Präsidenten des Europäischen Parlaments und des Präsidenten der Europäischen Zentralbank. Und bei der Besetzung dieser Spitzenämter gilt es, wie alle Erfahrungen lehren, eine Vielzahl von Kriterien zu beachten. Dazu gehört die Berücksichtigung alter und neuerer, größerer und kleinerer, westlicher, östlicher, südlicher und nördlicher Mitgliedstaaten. Außerdem ist die Vertretung der größeren europäischen politischen Parteien zu berücksichtigen. Nicht zuletzt ist auch die angemessene Beteiligung von Frauen bei der Besetzung von Ämtern entscheidend. Diese Gemengelage erfordert - einer vielfach geübten Praxis in der EU folgend - das Schnüren eines Pakets. Aber unter - nach dem Brexit - 27 Staaten ist es ungleich schwieriger, einen „package deal“ zu erreichen, als bei geringerer Mitgliederzahl.

Am 2. Juli 2019 billigten die Mitglieder des Europäischen Rates, nach mehreren mühsamen Verhandlungsetappen, die folgende Paketlösung: Die deutsche Verteidigungsministerin Ursula von der Leyen (CDU60 und EVP) wird dem Europäischen Parlament als Kandidatin für das Amt der Präsidentin der Kommission vorgeschlagen $;{ }^{61}$ der spanische Minis-

60 Christlich Demokratische Union.

61 Die Entscheidung erfolgte einstimmig bei Enthaltung der deutschen Bundeskanzlerin Angela Merkel (CDU). Die SPD, ihr Partner in der Regierung der Großen Koalition, hatte diese Personalentscheidung kritisiert und damit ein Votum der Bundeskanzlerin zugunsten von der Leyens verhindert. 
ter für Äußeres, EU und Entwicklungshilfe Josep Borrell (PSOE und PES) ${ }^{62}$ soll das Amt des Hohen Vertreters übernehmen; der seit Dezember 2018 nur noch geschäftsführende Ministerpräsident Belgiens Charles Michel (MR ${ }^{63}$ und ALDE) wird Präsident des Europäischen Rates; ${ }^{64}$ die Französin Christine Lagarde, Präsidentin des Internationalen Währungsfonds, soll Mario Draghi im Amt des Präsidenten der Europäischen Zentralbank folgen; das Amt des Präsidenten des Europäischen Parlaments soll nach den Vorstellungen des Europäischen Rates in den ersten zweieinhalb Jahren der Wahlperiode ein Mitglied der S\&D-Fraktion übernehmen, dem für die zweite 2,5-Jahres-Phase ein Mitglied der EVPFraktion - voraussichtlich Weber - folgen soll.

Am 3. Juli 2019 stand im neu konstituierten Europäischen Parlament die Wahl seines Präsidenten auf der Tagesordnung. Im zweiten Wahlgang erhielt der Italiener David Sassoli (PD ${ }^{65}$ und S\&D-Fraktion) 345 Stimmen und war damit für einen Zeitraum von zweieinhalb Jahren als Präsident gewählt. 160 Stimmen wurden für den Tschechen Jan Zahradil (ODS und EKR-Fraktion) abgegeben, 119 Stimmen für die Deutsche Keller (Bündnis 90/Die Grünen und Fraktion Grüne/EFA) und 43 Stimmen für die Spanierin Sira Rego (UP ${ }^{66}$ und GUE/NGL-Fraktion).

Aus der größten Fraktion, der EVP, gab es - im Einklang mit der Paketlösung des Europäischen Rates vom Vortag - keinen Kandidaten. Außerdem wurden die 14 Vizepräsidenten gewählt: Fünf stellt zukünftig die EVP-Fraktion, drei die S\&D-Fraktion, je zwei kommen aus der RE-Fraktion und der Fraktion Grüne/EFA, einer aus der GUE/NGLFraktion und einer aus der Gruppe der fraktionslosen Abgeordneten.

Für Mitte Juli 2019 ist im Europäischen Parlament die Wahl ins Amt des Präsidenten der Kommission vorgesehen. Die vom Europäischen Rat für dieses Amt vorgeschlagene Kandidatin von der Leyen hat unmittelbar nach ihrer Nominierung damit begonnen, mit den Fraktionen des Europäischen Parlaments in Kontakt zu treten und um Zustimmung zu ihrer Kandidatur zu werben. Dabei wird es um Inhalte künftiger EU-Politik gehen und sicherlich auch um politisch-institutionelle Fragen des Verhältnisses der drei EU-Organe (Kommission, Parlament und Rat/Europäischer Rat) zueinander. Zu diesen Fragen gehört auch, ob das Spitzenkandidaten-Verfahren künftig noch angewendet werden kann und soll. ${ }^{67}$ Ganz unabhängig davon, wie die Antwort auf diese Frage ausfallen wird, hat der Vorgang der Bemühungen um ein Personal-Tableau erneut bestätigt, dass das Gewicht des Europäischen Rates stärker geworden ist.

62 Er hatte früher das Amt des Präsidenten des Europäischen Parlaments inne.

63 Mouvement Réformateur (Reformbewegung).

64 Am 2. Juli 2019 erfolgte seine Wahl durch den Europäischen Rat (entsprechend den Bestimmungen des Art. 15 Abs. 5 EUV), zunächst für einen Zeitraum von zweieinhalb Jahren ab dem 1. Dezember 2019 (eine einmalige Wiederwahl ist möglich).

65 Partito Democratico (Demokratische Partei).

66 Unidos Podemos („Vereint können wir“).

67 Berthold Rittberger hatte in einem Zeitungsbeitrag vor der schließlich erfolgten Paket-Entscheidung des Europäischen Rates vehement für die Beachtung des Verfahrens plädiert („Rettet die Spitzenkandidaten!“, in: Frankfurter Allgemeine Zeitung, 26. Juni 2019); seine Argumente gelten zweifellos auch für die Beibehaltung des Verfahrens. Der bald aus dem Amt scheidende Kommissionspräsident Juncker, der 2014 als damaliger „Spitzenkandidat" angetreten war, gibt dem Spitzenkandidaten-Verfahren nach der Entscheidung des Europäischen Rates keine Chancen mehr; er wird mit der Aussage zitiert, er sei der erste und zugleich letzte Spitzenkandidat, in: Frankfurter Allgemeine Zeitung, 6. Juli 2019. 
Die Abgeordneten und die Fraktionen im Europäischen Parlament stehen vor der Frage, ob sie von der Leyen mit ausreichender Mehrheit (d.h. mit der absoluten Mehrheit der Mitglieder, nicht nur der Anwesenden und Abstimmenden) ins Amt der Kommissionspräsidentin wählen oder ob sie zu einer Eskalation ihres Konflikts mit dem Europäischen Rat bereit und entschlossen sind. Letzteres würde die EU insofern in eine Krise stürzen, als dann ihre Handlungsfähigkeit (zunächst jedenfalls) beeinträchtigt und bedroht wäre. Es erscheint unwahrscheinlich, dass eine Mehrheit im Europäischen Parlament dazu bereit ist. ${ }^{68}$

68 Am 16. Juli 2019, also eine Woche nach Fertigstellung des Beitrags, wurde die vom Europäischen Rat einstimmig (bei Stimmenthaltung von Bundeskanzlerin Merkel) als Kandidatin vorgeschlagene von der Leyen im Europäischen Parlament mit 383 Stimmen (bei 327 Gegenstimmen, 22 Enthaltungen und einer ungültigen Stimme) zur neuen Präsidentin der Europäischen Kommission für die Wahlperiode 2019-2024 gewählt. Es war eine knappe Entscheidung: Von der Leyen erhielt neun Stimmen mehr als die mindestens erforderlichen 374. Obwohl es eine geheime Wahl war, kann als sicher gelten, dass die meisten Fraktionen nicht einheitlich abgestimmt haben. Gruppen von Abgeordneten hatten im Vorfeld der Wahl ihre Entscheidung (für oder gegen den Personalvorschlag des Europäischen Rates) öffentlich begründet. Für einen (nicht bezifferbaren) Teil der Neinstimmen war die Nichtbeachtung des Spitzenkandidaten-Verfahrens (und des expliziten Bekenntnisses einer Mehrheit der Abgeordneten zu diesem Verfahren) der Hauptgrund für ihr Votum, mit dem sie eine Eskalation des institutionellen Konflikts zwischen dem Europäischen Parlament und dem Europäischen Rat bewusst in Kauf genommen hätten. Mit der Wahlentscheidung vom 16. Juli war diese Option zunächst gegenstandslos geworden.

Die Wahl von der Leyens ist nur ein erster - wenngleich besonders wichtiger - Schritt auf dem Weg zur Bildung der neuen Europäischen Kommission, bei dem das Europäische Parlament (wie die gewählte Kommissionspräsidentin) wichtige Mitwirkungsrechte hat. Die von den Regierungen der Mitgliedstaaten als Mitglieder der neuen Kommission vorgeschlagenen Kandidatinnen und Kandidaten (werden es mit Blick auf die auch von von der Leyen geforderte Geschlechter-Parität jeweils zwei Namen sein?) werden sich im Europäischen Parlament (in den jeweils zuständigen Fachausschüssen) Anhörungen stellen, wobei nicht auszuschließen ist, dass - wie bereits bei früheren Verfahren zur Bildung der Kommission - gegen einzelne der Vorgeschlagenen mehrheitlich Einwände vorgetragen werden und die betreffende Regierung alternative Vorschläge machen muss. Dieser Prozess der Bildung der Kommission ist nicht zuletzt auch ein Test für das Zusammenwirken von gewählter Kommissionspräsidentin und einer Mehrheit des Europäischen Parlaments - ganz unabhängig von der Entscheidung der Abgeordneten bei der Wahl von der Leyens am 16. Juli. Am Ende des Prozesses steht ein Votum des Europäischen Parlaments zum gesamten Kommissionskollegium (vorgesehen für die Sitzungswoche vom 21. bis 24. Oktober) - sicherlich kein Anlass für einen neuen institutionellen Konflikt. Und dann beginnt, was man die Sacharbeit der neunten Wahlperiode nennen kann, bei der unter anderem zu beobachten sein wird, wie die Fraktionen im Europäischen Parlament mit den neuen politischen Konstellationen umgehen werden und was das für die Wahrnehmung der Rolle des Europäischen Parlaments im EU-Entscheidungsprozess bedeutet. 